\title{
ASPECTOS DA GESTÃO DE DESIGN ABORDADOS EM ARTIGOS: uma análise bibliométrica
}

\author{
Giana Carli Lorenzini \\ Lund University \\ giana.lorenzini@gmail.com \\ Cláudia de Souza Libânio \\ PPGEP/UFRGS, UniRitter, DEIS/UFCSPA \\ claudiasl@ufcspa.edu.br
Fernando Gonçalves Amaral
PPGEP/UFRGS
amaral@producao.ufrgs.br

Resumo: A evolução de estudos sobre Gestão de Design é apontada por pesquisadores da área como crescente, com oportunidades vislumbradas em múltiplos exemplos e modelos identificados na literatura no que tange a propor limites coesos para esta disciplina. A fim de suprir tal demanda, este trabalho objetiva realizar uma revisão de literatura por análise bibliométrica acerca do tema Gestão de Design, mapeando o estado da arte do tema em questão. A pesquisa foi realizada na World Wide Web, com busca pela palavra-chave Design Management através do banco de dados Web of Science. Os dados bibliométricos foram extraídos com suporte de softwares específicos e tabulados para comparação. Os resultados encontrados evidenciam o estado da arte e a evolução da pesquisa sobre o tema em questão.

Palavras-chave: análise bibliométrica; estado da arte; gestão de design; revisão de literatura.

\begin{abstract}
The evolution of studies about Design Management is cited as a growing field of research with opportunities envisioned in multiple examples and models from the literature regarding the proposed limits for this cohesive discipline. To achieve this demand, this paper aims to carry out a literature review by bibliometric analysis about the theme Design Management, mapping the state of the art. The study was conducted on the World Wide Web, with search by the keyword Design Management through the database Web of Science. The bibliometric data were extracted with support of specific software and tabulated for comparison. The results show the state of art and the evolution of research on the topic in question.
\end{abstract}


Keywords: bibliometric analysis; state of the art; design management; literature review.

\section{INTRODUÇÃO}

As primeiras discussões sobre o tema Gestão de Design (GD) ou Design Management (DM) iniciaram-se na Grã-Bretanha. Farr (1966) observou uma nova função entre os meios de gestão: o gestor de design. Este novo profissional teria a missão de proporcionar uma comunicação mais efetiva entre os designers externos e as organizações. Em 1975, Bill Hannon e o Massachusetts College of Art fundaram o DMI (Design Management Institute), em Boston nos Estados Unidos (BORJA DE MOZOTA, 2003), sendo este Instituto uma importante referência na área até os dias de hoje.

Ao longo das últimas décadas, a GD vem sendo discutida mundialmente como um fator-chave para o sucesso corporativo. Estudos internacionais de autores como Gorb (1990), Bruce et al. (1999), Borja de Mozota (2003), Best (2006), entre outros, se tornaram referências no assunto. Além destes, merecem destaque os trabalhos elaborados por institutos de pesquisa como: o Design Management Institute - DMI nos Estados Unidos; International Council of Societies of Industrial Design - ICSID no Canadá, Centro Português de Design - CPD em Portugal, o Centro de Design da Dinamarca - CDD; e o Instituto Europeu de Design - IED na Itália. Somado às pesquisas internacionais, verifica-se também um maior interesse de empresas em entender e estruturar o gerenciamento dos processos de design, a exemplo dos estudos realizados por Borja de Mozota e Kim (2009); Neumeier (2008); Lojacono e Zaccai (2004); Beverland e Farrelly (2007).

De modo geral, GD pode ser entendida como a implantação do design em um programa formal de atividades na organização, reconhecendo sua relevância para os objetivos de longo prazo da companhia, coordenando os seus recursos em todos os níveis da atividade corporativa, almejando alcançar os objetivos da empresa (BORJA DE MOZOTA, 2003). Os autores citados entendem a Gestão de Design com uma ação mais ampla e global na companhia, posicionando o design num patamar maior de responsabilidades na corporação. Corroborando o conceito de GD atuando em nível mais amplo e global na companhia, Spoladore e Demarchi (2004) destacam a necessidade de condicionar recursos e métodos, e assim organizar o ambiente de maneira a propiciar o surgimento de novos produtos. Avendaño (2005) destaca a interação entre os setores da empresa possibilitando uma participação mais ativa do design nas decisões sobre produtos. Nesse sentido, GD pode ser uma ferramenta estratégica capaz de estabelecer a inserção adequada do design na empresa através do gerenciamento de recursos humanos e materiais da organização, integrando áreas especializadas à alta administração, desde a concepção de um projeto até seu lançamento no mercado (RODRIGUES, 2005). Visualizando a Gestão de Design como uma ponte entre o design e o negócio, McBride (2007) defendeu a liderança focada no design, que vai além da estratégia, transformando experiências, companhias e oportunidades. Cria-se, assim, espaço para a liderança através do design e a permanência de uma cultura de design de forma holística nas organizações (BEVERLAND; FARRELLY, 2007; GLOPPEN, 2009; NAM; JUNG, 2008; HARGADON, 2005).

O DMI (2010) entende que existem duas maneiras de compreender a GD. Em nível geral, entende a GD como a face de negócio do design, pois engloba os processos 
em curso, as decisões de negócios bem como as estratégias que permitem a inovação e a criação de design efetivo em produtos, serviços, comunicações, ambientes e marcas, melhorando a qualidade de vida e proporcionando sucesso organizacional. Já em nível mais específico, salienta que a GD visa unir design, inovação, tecnologia, gestão e clientes para oferecer vantagens competitivas através de três linhas base (econômica, social/cultural e fatores ambientais), promovendo a colaboração e a sinergia entre design e negócios e assim, melhorando a eficácia do design (DMI, 2010). Outro instituto de pesquisa atuante na área, o Design Council (2010) define a GD como sendo uma atividade total de design, desde a implementação e organização de todo o processo de desenvolvimento de novos produtos e serviços até a administração e o alcance de um melhor desempenho da empresa.

Além da visão isolada de autores e instituições sobre as definições, pode-se propor a elaboração de um panorama capaz de mapear o estado da arte sobre o tema GD, contrapondo os avanços científicos no Brasil e no mundo. Para atingir esse objetivo, o presente estudo aplica o método bibliométrico associado a recursos de busca online.

\section{PROCEDIMENTOS METODOLÓGICOS}

A partir da proposta deste estudo, optou-se por realizar uma revisão de literatura, orientada por princípios da análise bibliométrica de natureza aplicada, com objetivos de caráter exploratório. Conforme definido por Vanti (2002), a avaliação sistematizada sobre um determinado ramo de conhecimento pode mostrar de que forma esse saber vem se desenvolvendo e de que forma tem se dado suas contribuições no meio em que está inserido. Dessa forma, a autora define que esse tipo de procedimento de análise ajuda a identificar núcleos de pesquisa e tendências de publicação; estuda a dispersão e a obsolescência da literatura científica; prevê a produtividade de autores individuais, organizações e países; analisa processos de citação e co-citação; avalia os aspectos estatísticos da linguagem, das palavras e das frases; mede o crescimento de determinadas áreas e o surgimento de novos temas.

No presente estudo, alguns desses pontos foram evidenciados, como a construção de conceitos, identificados a partir das palavras-chave e sua composição visual em forma de 'nuvens de palavras'. Foram seguidos procedimentos e estruturação metodológica semelhante à aplicada por Marzagão et al. (2010).

$\mathrm{Na}$ área de design e de GD, não são muito recorrentes as análises bibliométricas, com a percepção recente sobre a necessidade de buscar recorrer a uma consolidação de terminologias, impacto e evolução do que tem sido produzido. Chai e Xiao (2011) realizaram estudo semelhante a esta proposta, tendo como objetivo investigar temas centrais da pesquisa em design pela análise de citações de trabalhos no periódico 'Design Studies', apontando as principais tendências e evoluções na área.

\subsection{Coleta de dados}

Para coleta de dados, procedeu-se com as definições de cada etapa de pesquisa (Quadro 1), a exemplo de estudos semelhantes realizados por Sampaio e Mancini (2007) e Brereton et al. (2007). 
Quadro 1 - Etapas para a análise bibliométrica.

\begin{tabular}{|l|l|}
\hline $\begin{array}{l}\text { Etapas para a Revisão de Literatura com } \\
\text { Análise Bibliométrica }\end{array}$ & Etapas da pesquisa sobre Gestão de Design \\
\hline 1) Definir a pergunta científica & $\begin{array}{l}\text { Qual o atual estágio de produção intelectual sobre o } \\
\text { tema Gestão de Design? }\end{array}$ \\
\hline $\begin{array}{l}\text { 2) Identificar as bases de dados a serem } \\
\text { consultadas, definir palavras-chave e e } \\
\text { estratégias de busca }\end{array}$ & $\begin{array}{l}\text { Para coleta dos estudos em questão foi utilizada a World } \\
\text { Wide Web, na qual se recorreu ao sistema eletrônico } \\
\text { Web of Knowledge. Dentro desse portal, optou-se por } \\
\text { realizar as buscas na base de dados Web of Science, na } \\
\text { qual a busca foi conduzida através da pesquisa pelo } \\
\text { termo-chave design management. }\end{array}$ \\
\hline
\end{tabular}

4) Estabelecer critérios de inclusão/ exclusão - Artigo deveria apresentar: GD como estratégia de de artigos negócio e área de conhecimento no negócio, considerando-se artigos sobre design relacionado aos processos de desenvolvimento de produto (bens ou serviços) em diferentes ramos setoriais; modelos teóricos sobre GD; estudos empíricos sobre o papel estratégico do design nas empresas e a sua relação com sistemas organizacionais, profissionais especializados e desenvolvimento de competências na área:

- Artigos relacionados às áreas, selecionáveis nos mecanismos de busca: management; engineering industrial; engineering multidisciplinary; engineering manufacturing; operations research management science; business; multidisciplinary sciences; planning development; social issues; communication; education scientific disciplines.

4) Buscar em bases de dados escolhidas e Foram feitas buscas sobre os artigos conduzidas por três com base em estratégia(s) definida(s) pesquisadores independentes.

5) Comparar as buscas dos examinadores e As buscas dos três pesquisadores foram comparadas, definir a seleção de artigos apresentando resultados idênticos, totalizando em 95 artigos encontrados.

6) Aplicar os critérios na seleção dos artigos e justificar possíveis exclusões

Considerando o critério de busca sobre o conteúdo, a qual se procedeu pela leitura de título e resumo, foram eliminados 58 artigos, restando 35 artigos para leitura integral.

7) Analisar criticamente e avaliar todos os As informações dos trabalhos foram organizadas e estudos incluídos na revisão

tabeladas, de maneira que fosse possível desenvolver comparações e análises.

8) Preparar um resumo crítico, sintetizando Um resumo crítico em formato de tabela eletrônica foi as informações disponibilizadas pelos artigos realizado, onde as informações de cada trabalho que foram incluídos na revisão julgadas relevantes para este estudo foram elencadas, como número de identificação do artigo; base de origem; título em inglês; autor(es); periódico (journal); ano de publicação; resumo; ramo setorial; objetivo; método; principais resultados/conclusão.

9) Quantificação e qualificação dos dados Os dados foram quantificados a partir do suporte de recursos de análise disponível na base Web of Science. Além disso, foi feito um reporte de citações e co-citações com a utilização dos softwares Sitkis, UCINET e Histcite a fim de verificar a relação entre os estudos.

10) Apresentar uma conclusão, informando a A partir da análise das informações contidas nos 35 evidência sobre os efeitos da intervenção artigos foi possível mapear o atual estágio da produção intelectual sobre o tema Gestão de Design.

Fonte: Adaptado de Sampaio e Mancini (2007); Brereton et al. (2007). 
A definição dos critérios de busca booleana e de inclusão/ exclusão aplicados foi complementada a partir da leitura da revisão realizada por Becheikh et al. (2006). Inicialmente, foram definidas etapas de investigação com a finalidade de orientar a pesquisa dentro de critérios pré-estabelecidos, em conformidade com o objetivo proposto.

\subsection{Procedimentos para análise de dados}

Os dados foram organizados a partir da sua tabulação em planilhas eletrônicas e subdivididos em: número de identificação do artigo; base de origem; título em inglês; autor(es); periódico (journal); ano de publicação; resumo; ramo setorial; objetivo; método; principais resultados/conclusão.

Essa organização preliminar possibilitou o resumo crítico dos artigos e a posterior composição dos dados em gráficos e tabelas, os quais embasam também a discussão a partir de objetivos, métodos, resultados e conclusões encontrados.

\section{RESULTADOS E DISCUSSÃO}

Para apresentação dos resultados, considerou-se importante fazer a tabulação dos seguintes dados dos artigos: tipologia e ano de publicação; centros de pesquisa e locais de produção; palavras-chave e análise de co-citações.

\subsection{Tipologia e ano de publicação}

Foram encontrados 35 artigos desenvolvidos em universidades como MIT, Helsinki University of Technology, Politecnico di Milano, University of Newcastle etc. porém com grande distribuição entre diversas universidades identificadas. Com relação ao ano de publicação, nota-se um crescimento com o passar dos anos, principalmente entre 2006 e 2008.

O crescimento, porém, não é contínuo, sendo alterado com períodos de redução do volume de publicações. Entre 2002 e 2003, por exemplo, o número de artigo caiu. De maneira geral, nota-se um crescimento no número de publicação a partir do ano 2000. Este é um ponto interessante, pois demonstra a evolução e interesse pelas pesquisas sobre o tema em questão, corroborando estudo anterior de Libânio e Amaral (2011).

\subsection{Centros de pesquisa e locais de publicação}

Foi observada uma grande dispersão entre as universidades, com destaque para as européias, com destaque para Reino Unido (Glasgow Caledonian University, Manchester School of Management, University of Hull, University of Newcastle, University of Aberdeen, University of Leeds, University of Strathclyde, The Open University, University of Warwick, University of Strathclyde, University of Westminster) e as norte-americanas, originárias dos EUA (University of California, Massachusetts Institute of Technology, University of Wisconsin, Bowling Green State University e Wayne State University) que apresentaram maior quantidade de publicações.

O volume de produções internacionais Europa e América do Norte condiz com - local de surgimento das primeiras escolas e instituições de pesquisa voltadas à Gestão de Design, tal qual apontado por Farr (1966) e Borja de Mozota (2003). Chama a atenção a presença de artigos provenientes da China e Singapura, que parecem evidenciar um novo polo de produção da área. Em relação às regiões de publicação, destacam-se Europa (Reino Unido, Espanha, Finlândia, Holanda, América do Norte). 


\subsection{Palavras-chave}

Por serem publicações internacionais e, portanto, publicados no idioma inglês, os termos precisam ser traduzidos com alguma diferença ocasionada por esse diferencial entre línguas. Ainda assim, verificou-se que a palavra-chave design management (gestão de design; gestão do design) apareceu 15 vezes, seguida de product development (desenvolvimento de produto) e product design (design de produto). Os termos case study/ case studies (estudo de caso/ estudos de caso); industrial design (desenho industrial); product design management (gerenciamento de design de produto); design strategy (estratégia de design); design process (processo de design); modelling (modelagem) e communication (comunicação) apareceram duas vezes cada. Em doze artigos não houve definição de palavra-chave.

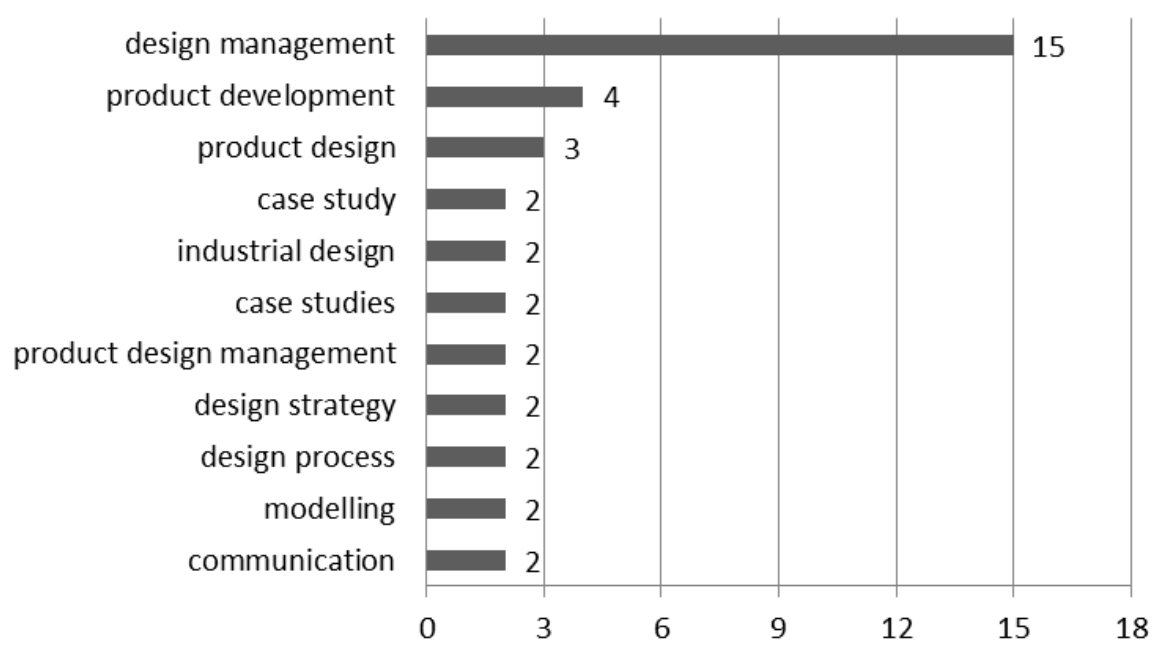

Figura 1 - Palavras-chave versus quantidade nos artigos

Fonte: Elaborado pelos autores, com base na pesquisa realizada.

A Figura 1 mostra o comparativo de termos mais presentes nos artigos, seguidos da ilustração (Figura 2) na qual se evidenciou pelo tamanho da palavra a quantidade de vezes em que esta apareceu - sendo que quanto maior a palavra, maior a quantidade de vezes em que foi identificada.

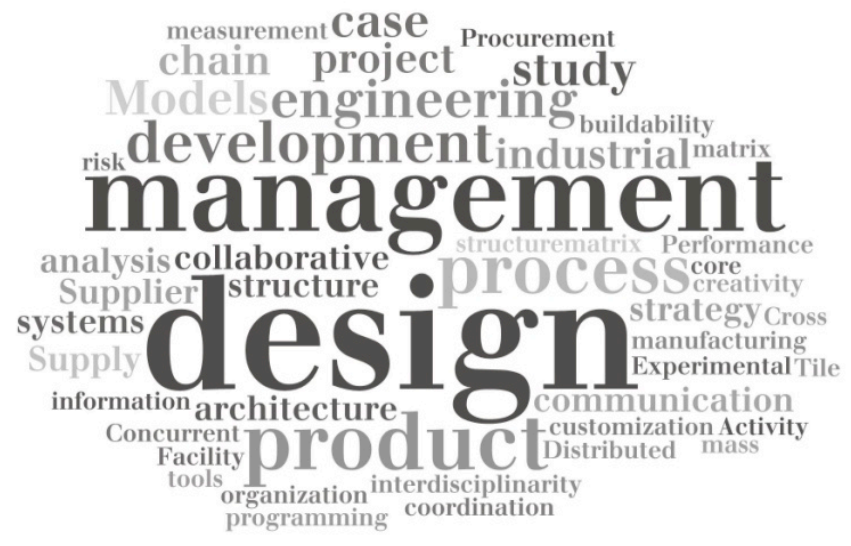

Figura 2 - Nuvem com títulos e palavras-chave dos artigos

Fonte: Elaborado pelos autores, com base na pesquisa realizada.

Os estudos apresentaram palavras-chave mais relacionadas ao domínio do desenho industrial e ao projeto de produto em estudos de caso e em estudos sobre os 
processos de design. No Brasil, tem sido reforçada a aproximação do design da estratégia da empresa e de seu avanço competitivo. No ambiente internacional, trabalha-se a ideia de projeto abrangente a áreas de domínio industrial, com foco em engenharia e processos de manufatura. Discute-se ainda a integração do design em cadeias mais complexas com parceiros de negócio que tem o design como core competence (Bruce; Morris, 1994; Bonjour; Micaelli, 2010; Ivory et al., 2003; McCutcheon et al., 1997; Roy; Potter, 1993; Twigg, 1998) e também a construção de modelos e matrizes que auxiliem na busca por melhor performance através do design (Ahamadi et al., 2001; Chiva-Gomez, 2004, Chua et al., 2003; Manzini et al., 2008; O'Donnell; Duffy, 2002). Somado a isso, os artigos internacionais destacam a importância de como medir o desempenho do design e seu impacto na empresa (Chiva; Alegre, 2009; Sheu, Chen, 2007; Wong et al., 2009).

\subsection{Análise de Co-citações}

Assim como Chai e Xiao (2011), neste estudo também foram encontrados agrupamentos de co-citações de autores e suas pesquisas a partir do conjunto de referências dos 35 artigos internacionais selecionados previamente, indicando o compartilhamento de ideias e temas entre as publicações. A Figura 3 mostra a formação de três grandes grupos (Grupos A, B e C), seguidos de um grupo menor (Grupo D) e de alguns outros autores com menos vinculações. As setas indicam a correlação entre um ponto e outro, podendo estar no mesmo grupo ou em grupos distantes. A formação de grupos é aqui estabelecida pela proximidade e concentração de setas ao redor de um mesmo nó.

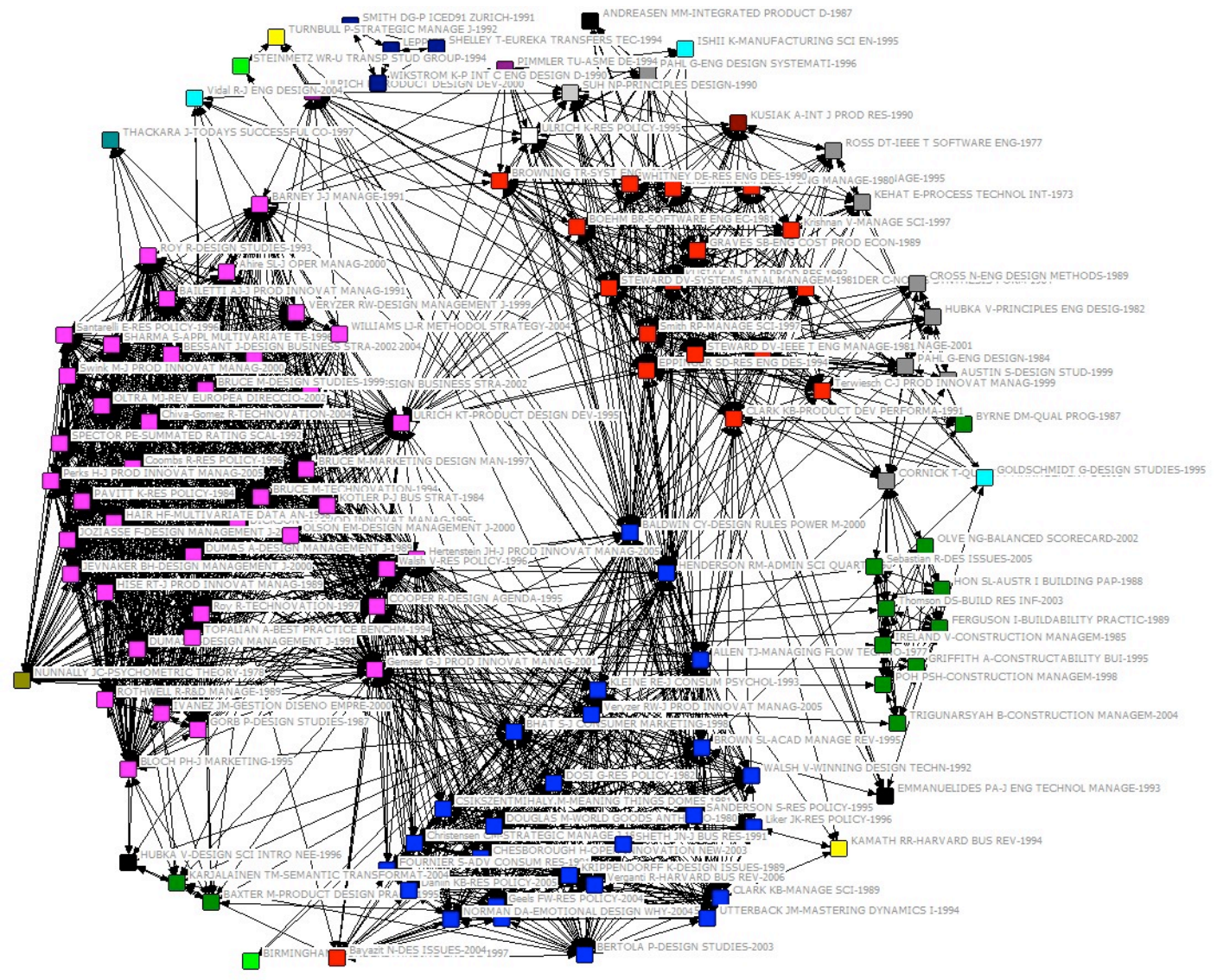

Figura 3 - Mapa de co-citações para a pesquisa de Gestão de Design Fonte: Web of Science, UCINET 
O Grupo A, evidenciado à esquerda na cor magenta, foi o que manteve mais citações, além disso foi o que melhor manteve centralizado o tema de Gestão de Design nos critérios desta pesquisa. Nas extremidades, fora do centro do grupo, destacam-se trabalhos como o de Roy (1993), Hertenstein (2005), Walsh (1996). O trabalho de Ulrich e Eppinger (1995) também aparece fora do grupo, porém sua posição demonstra um polo para o qual convergem muito artigos do Grupo A. Ao mesmo tempo, esses autores conectam o Grupo A, focado em Gestão de Design, com o Grupo B (em vermelho) - em que se encontram autores e publicações vinculados a uma visão de produto mais sistêmica, analítica e próxima da engenharia. A quantidade de correlações é menor, com autores do Grupo B como Eppinger (1994) e Clark (1991) fazendo a correlação com o Grupo C. No Grupo C (em azul escuro), as co-citações envolvem publicações que abordam o design aproximado de conceitos de inovação como visto em Bertola e Teixeira, 2003 e Chesbrough (2003). Também estão presentes trabalhos relacionados a aspectos de comportamento do consumidor e ao design emocional. Por fim, identificou-se um pequeno grupo em formação - Grupo D - em que se evidencia a concentração na temática de design relacionado à construção e sua administração (verde escuro).

De um modo geral, as pesquisas dos Grupos B e C são mais antigas e servem como cerne para os estudos posteriores, principalmente os trabalhos mais recentes do Grupo A, no qual se verificou uma grande concentração de correlações. A presença das correlações entre esses grupos mostra a evolução na área de GD, para a qual são também delineadas as origens a partir do desenvolvimento dos processos de produto e sua conexão com os conceitos de inovação nas empresas. O grande número de correlações do Grupo A também aponta para um fortalecimento do campo de pesquisa em GD, com uma grande quantidade de pesquisas recentes. Conforme descrito por Vanti (2002), a avaliação sistematizada mostra o desenvolvimento de um saber e suas contribuições, o que ficou bastante evidente no mapa de agrupamento de co-citações (Figura 3), percebeu-se também a dispersão de alguns temas mais afastados (a exemplo do Grupo D) e a formação de novos núcleos de pesquisa (Grupo A).

Outro aspecto a observado foram as citações e co-citações de alguns artigos específicos. No artigo de Eppinger (1994), por exemplo, chegam seis citações, provenientes de O'Donnell (2002); Whitfield (2002); Pektas (2006). Observa-se que de um artigo mais recente de Bonjour (2010) parte uma citação direta para Eppinger (1994) e uma citação em direção a Browning (2002), que segue para Ahmadi (2001) e deste, finalmente, para Eppinger (1994). Neste caso, se nota que mesmo produções mais recentes ainda são impactas pelo trabalho de Eppinger (1994).

Em relação ao trabalho de Bruce (1994) verificou-se um caso semelhante, porém com um maior número de entrelaçamento de nós ao invés da citação direta ao trabalho. Autores que citam Bruce (1994) citam também Dickson (1995), mostrando proximidade pelo interesse em ambos os estudos. Novamente, se observa que estudos mais recentes (Chiva; Alegre, 2007; Chiva, 2009) recorreram a estudos anteriores em mais de uma década, o indica o embasamento de conceitos novos sobre GD a partir autores referenciais de design e desenvolvimento de produto. Autores com um volume maior de produção na área também acabam por influenciar nas relações de citações, a exemplo de Chiva, que recorreu aos mesmos autores em suas pesquisas. 


\section{CONCLUSÃO}

Este artigo apresentou uma análise bibliométrica acerca do tema GD. Com isto, foi possível mapear a produção intelectual já desenvolvida que abordasse o tema , evidenciando oportunidades de pesquisa na área.

Baseado no conjunto de informações coletadas, pôde-se concluir que a GD é, cada vez mais, entendida de forma multidisciplinar, incentivando um intercâmbio de conhecimentos e reforçando uma visão pluralista. Percebe-se ainda um crescente de publicações bem como uma evolução gradativa no entendimento da área em questão, evidenciando um maior interesse e oportunidades de pesquisa no estudo da gestão de design. Entende-se ser positiva a evolução de estudos na área, assim como a multidisciplinaridade dos centros de pesquisa que estudam o tema, possibilitando uma maior integração de conceitos e enfatizando o caráter multidisciplinar e integrador de GD. Entretanto, observa-se o debate de certos aspectos de pesquisa primeiramente no exterior e posteriormente no Brasil. Esta percepção corrobora a visão de Libânio e Amaral (2013) acerca, por exemplo, do estudo sobre o papel do profissional de design na gestão de design. Segundo os autores, pode-se concluir que essa diferença resulta do meio em que o profissional vive, mas em grande parte advém da formação acadêmica desse profissional e do perfil pessoal de cada indivíduo.

Dentre as limitações da pesquisa, está o conjunto de apenas 35 artigos, os quais não permitem uma generalização de resultados sobre GD. Ainda assim, os estudos revisados são relevantes e futuras pesquisas podem ampliar as buscas em novas análises quantitativas. A pesquisa realizada tem a limitação de um conjunto de 35 artigos de determinados periódicos internacionais, verificados a partir de uma base de dados específica, não sendo possível a aplicação de seus resultados bem como de suas conclusões na totalidade de estudos sobre gestão de design. Portanto, sugere-se ampliar ainda mais o estudo e o desenvolvimento de pesquisas futuras em estudos semelhantes.

\section{REFERÊNCIAS}

AHMADI, R.; ROEMER, T. A.; WANG, R.H. Structuring product development processes. European Journal of Operational Research, v.130, n.3, p. 539-558, 2001.

AVENDAÑO, L.E.C. Resgate do protagonismo do desenhista industrial por meio da gestão do design. Revista do Programa de Pós-Graduação em Arquitetura e Urbanismo da FAUUSP/USP, São Paulo: USP, 2005.

BECHEIKH, N., LANDRY, R., AMARA, N. Lessons from innovation empirical studies in the manufacturing sector: a systematic review of literature from 1993 - 2003. Technovation, 26, 644-664, 2006.

BERTOLA, P.; TEIXEIRA, J.C. Design as a knowledge agent: How design as a knowledge process is embedded into organizations to foster innovation. Design Studies, v. 24, n. 2, p. 181-194, 2003.

BEST, K. Design Management: managing design strategy, process and implementation. Lausanne: AVA, 2006.

BEVERLAND, M., FARRELLY, F. What does it mean to be design-led? Design Management Review, 18 (4), 10-17, 2007. 
BONJOUR, E.; MICAELLI, J.P. Design Core Competence Diagnosis: A Case From the Automotive Industry. IEEE Transactions on Engineering Management, v.57, n.2, p.323337, 2010.

BORJA DE MOZOTA, B. Design Management: Using Design to Build Brand Value and Corporate Innovation. New York: Allworth, 2003.

BORJA DE MOZOTA, B., KIM, B. Managing design as a core competency: lessons from Korea. Design Management Review, 20 (2), 66-76, 2009.

BRERETON, P., KITCHENHAM, B. A., BUDGEN, D., TURNER, M.; KHALIL, M. Lessons from applying the systematic literature review process within the software engineering domain. The Journal of Systems and Software, 80 (4), 571- 583, 2007.

BROWNING, T.R.; EPPINGER, S.D. Modeling impacts of process architecture on cost and schedule risk in product development. IEEE Transactions on Engineering Management, v.49, n.4, p.428-442, 2002.

BRUCE, M.; MORRIS, B. Managing external design professionals in the product development process. Technovation, v.14, n.9, p.585-599,1994.

BRUCE, M.; COOPER, R.; VAZQUEZ, D. Effective design management for small businesses. Design Studies, 1999.

CHAI, K., XIAO, X. Understanding design research: A bibliometric analysis of Design Studies (1996-2010). Design Studies, 2011.

CHIVA, R.; ALEGRE, J. Linking design management skills and design function organization: An empirical study of Spanish and Italian ceramic tile producers. Technovation, v.27, n.10, p.616-627, 2007.

CHIVA, R.; ALEGRE, J. Investment in Design and Firm Performance: The Mediating Role of Design Management. Journal of Product Innovation Management, v.26, n.4, p.424-440, 2009.

CHIVA-GOMEZ, R. Repercussions of complex adaptive systems on product design management. Technovation, v.24, n.9, p.707-71, 2004.

CHUA, D.K.H.; TYAGI, A.; LING, S.; BOK, S.H. Process-parameter-interface model for design management. Journal of Construction Engineering and Management-Asce, v.129, n.6, p.653-663, 2003.

DESIGN COUNCIL. Disponível em: http://www.designcouncil.org.uk Acesso em: jul. 2013.

DICKSON, P.; SCHNEIER, W.; LAWRENCE, P.; HYTRY, R. Managing Design in Small HighGrowth Companies. Journal of Product Innovation Management, v.12, n.5, p.40641,1995 .

DMI - Design Management Institute. Disponível em: http://www.dmi.org Acesso em: ago. 2013.

EPPINGER, S.D.; WHITNEY, D.E.; SMITH, R.P.; GEBALA, D. A. A model-based method for organizing tasks in product development. Research in Engineering Design - Theory Applications and Concurrent Engineering, v.6, n.1, p.1-13, 1994. 
FARR 1996 In: BORJA DE MOZOTA, Brigitte. Design Management: Using Design to Build Brand Value and Corporate Innovation. New York: Allworth, 2003.

GLOPPEN, J. Perspectives on design leadership and design thinking and how they relate to European service industries. Design Management Journal, v. 44, n. 1, 33-47, 2009.

GORB, P. Design Management. New York: Van Nostrand Reinhold, 1990.

HARGADON, A. Leading with vision: the design of new ventures. Design Management Review, 16 (1), 33-39, 2005.

HERTENSTEIN, J., PLATT, M., VERYZER, R. The impact of industrial design effectiveness on corporate financial performance. Journal of Product Innovation Management, $v$. 22, n. 1, p. 3-21, 2005.

HISTCITE - Bibliographic Analysis and Visualization Software. Disponível em: <http:// http://www.garfield.library.upenn.edu/histcomp/index.html>. Acesso em: nov. 2011.

IVORY, C.J.; THWAITES, A.T.; VAUGHAN, R. Shifting the goal posts for design management in capital goods projects: 'design for maintainability'. R\&D Management, v. 33, n.5, p.527-538, 2003.

LIBÂNIO, C. S. AMARAL, F. G. Aspectos da gestão de design abordados em dissertações e teses no Brasil: uma revisão sistemática. Revista Produção Online, v. 11, 565-594, 2011.

LIBÂNIO, C.S.; AMARAL, F.G. Design Professionals Involved in Design Management: Roles and Interactions in Different Scenarios: A Systematic Review. In: Chakrabarti, A.; Prakash, R.V. (eds.), ICoRD'13, Lecture Notes in Mechanical Engineering, DOI: 10.1007/978-81-322-1050-4_69, Springer India, 2013.

LOJACONO, G., ZACCAI, G. The evolution of the design-inspired enterprise. MIT Sloan Management Review, 45 (3), 75-79, 2004.

MANZINI, R.; GAMBERI, M.; GEBENNINI, E.; REGATTIERI, A. An integrated approach to the design and management of a supply chain system. International Journal of Advanced Manufacturing Technology, v.37; n.5-6, p.625-640, 2008.

MAZARGÃO, D.S.L., ERARIO, A.L., CARVALHO, M.M. Sistemas de indicadores de desempenho: revisão bibliográfica por métodos bibliométricos. Encontro Nacional de Engenharia de Produção, 30. Anais... São Carlos, SP, 2010.

MCBRIDE, M. Design Management: Future Forward. Design Management Review; no18-3, DMI: 2007.

MCCUTCHEON, D.M.; GRANT, R.A.; HARTLEY, J. Determinants of new product designers' satisfaction with suppliers' contributions. Journal of Engineering and Technology Management, v.14, n.3-4, p.273-290, 1997.

NAM, K., JUNG, M. Bottom-up design leadership as a strategic tool. Design Management Review, 19 (3), 59-67, 2008.

NEUMEIER, M. The designful company. Design Management Review, 19 (2), 10-15, 2008. 
O'DONNELL, F.J.; DUFFY, A.H.B. Modelling design development performance. International Journal of Operations \& Production Management, v.22, n.11, p.11981221, 2002.

PEKTAS, S.T.; PULTAR, M. Modelling detailed information flows in building design with the parameter-based design structure matrix. Design Studies, v.27, n.1, p.99122, 2006.

RODRIGUES, R.B. A atividade de gestão do design nas organizações: um estudo no pólo moveleiro de Santa Catarina. Dissertação de Mestrado, Biguaçu: Universidade do Vale do Itajaí, 2005.

ROY, R.; POTTER, S. Managing engineering design in complex supply chains. International Journal of Technology Management, v.12, n.4, p.403-420, 1993.

SAMPAIO, R.F; MANCINI, M.C. Estudos de revisão sistemática: um guia para síntese criteriosa da evidência científica. Revista Brasileira de Fisioterapia, São Carlos, v.11, n.1, 2007.

SHEU, D.D.; CHEN, D.R. Backward design and cross-functional design management. Computers \& Industrial Engineering, v.53, n.1, p.1-16, 2007.

SPOLADORE, R., DEMARCHI, A. A Gestão do Design no desenvolvimento de produtos na Indústria Digital. Anais do P\&D Design, São Paulo, SP, 2004.

TWIGG, D. Managing product development within a design chain. International Journal of Operations \& Production Management. v.18, n.5-6, p.508-524, 1998.

ULRICH, K.T., EPPINGER, S.D. Product Design and Development. Nova York: McGrawHill,1995.

VANTI, N.A.P. Da bibliometria à webometria: uma exploração conceitual dos mecanismos utilizados para medir o registro da informação e a difusão do conhecimento. Ci. Inf., v. 31, n. 2, p. 152-162, 2002.

WALSH, V. Design, innovation and the boundaries of the firm. Research Policy, v. 24, n. 4, p. 509-529, 1996.

WHITFIELD, R.I.; DUFFY, A.H.B.; COATES, G.; HILLS, W. Distributed design coordination. Research in Engineering Design-Theory Applications and Concurrent Engineering, v.13, n.4, p.243-252, 2002.

WONG, F.W.H.; LAM, P.T.I.; CHAN, E.H.W. Optimising design objectives using the Balanced Scorecard approach. Design Studies, v.3, n.4, p.369-392, 2009. 